\title{
Characteristics of leprosy diagnosed through the surveillance of contacts: a comparison with index cases in Rio de Janeiro, 1987-2010
}

\author{
Mariana de Andrea Hacker ${ }^{+}$, Nadia Cristina Duppre, José Augusto Costa Nery, \\ Anna Maria Sales, Euzenir Nunes Sarno
}

Laboratório de Hanseníase, Instituto Oswaldo Cruz-Fiocruz, Rio de Janeiro, RJ, Brasil

Contact surveillance is an important strategy to ensure effective early diagnosis and control of leprosy; passive detection may not be as efficient because it is directly tied to the ready availability of heath care services and health education campaigns. The aim of this study was to reinforce that contact surveillance is the most effective strategy for the control of leprosy. The analysed data were obtained from a cohort of contacts and cases diagnosed through a national referral service for leprosy. We analysed data from patients diagnosed between 1987-2010 at the Souza Araújo Ambulatory in Rio de Janeiro. Epidemiological characteristics of leprosy cases diagnosed through contact surveillance and characteristics of passively detected index cases were compared using a conditional logistic regression model. Cases diagnosed by contact surveillance were found earlier in the progression of the disease, resulting in less severe clinical presentations, lower levels of initial and final disability grades, lower initial and final bacterial indices and a lower prevalence of disease reaction. In this respect, contact surveillance proved to be an effective tertiary prevention strategy, indicating that active surveillance is especially important in areas of high endemicity, such as Brazil.

Key words: surveillance - contact - leprosy

Leprosy has remained an important public health care problem, despite the adoption of specific goals and strategies from the World Health Organization (WHO) to eliminate this disease across the globe. The annual number of cases detected has been declining worldwide; in 2003 , more than 514,000 cases were detected, while only 228,474 cases were reported in 2010. In Brazil, 34,894 new cases were detected in 2010. The global prevalence of leprosy was 192,246 cases in early 2011 (WHO 2011).

In addition to multi-drug therapy, the Brazilian leprosy control programme includes the early diagnosis of new cases, together with clinical examination and intradermal BCG vaccination of contacts considered most at risk for developing the disease (MS 2002). Studies have shown that once contacts have received the BCG vaccine (either the 1st or 2nd dose) and the primary patient has begun treatment, the risk of contracting the disease declines (Düppre et al. 2008).

When leprosy is either untreated or diagnosed late, there is a high probability that disabilities will occur. However, once leprosy is diagnosed, early treatment reduces the potential for developing disabilities, which are responsible for the stigma and discrimination that have been experienced by many leprosy patients throughout history (Mankar et al. 2011).

+ Corresponding author: marianah@ioc.fiocruz.br

Received 15 March 2012

Accepted 18 July 2012
Epidemiological surveillance of contacts is a highly efficient component of leprosy control. Actively searching for new cases is pivotal for early detection while simultaneously interrupting the chain of transmission and helping to prevent the disabilities that arise from untreated disease. The importance of contact surveillance in controlling the incidence of leprosy has been consistently highlighted by the Brazilian Ministry of Health (MS 2006).

The aim of this study was to compare the epidemiological characteristics of the cases diagnosed through contact surveillance with the characteristics of the passively detected cases. This comparison could yield information to improve leprosy prevention and control strategies in endemic areas of Brazil and help acquire subsidies to implement these strategies.

\section{PATIENTS, MATERIALS AND METHODS}

Selection of participants - The patients at the Souza Araújo Ambulatory (SAA) clinic are primarily from the metropolitan region of Rio de Janeiro, but also come from other cities in the state. Because the clinic is a national referral centre, coverage is not limited to any particular location. Most of the patients in the study $(70 \%)$ were referred from other health care institutions (both public and private); the remaining patients either arrived spontaneously (approximately 6\%) or were identified by contact surveillance (approximately 16\%). After the clinical, histopathological and bacteriological confirmations of leprosy were obtained, the patients began treatment and were monitored. The services of the clinic include outpatient dermatology, neurology, physiotherapy and health care education. Patient and contact information, including socio-economic, clinical and laboratory data, is recorded 
in the SAA clinical database. The database has been regularly and systematically maintained since 1987 .

All of the outpatients were classified by clinical examination and histopathology tests according to the Ridley and Jopling (1966) criteria. The spectral classification of leprosy is divided into two polar groups, namely tuberculoid (TT) and lepromatous (LL) and three intermediate types, namely borderline tuberculoid (BT), borderline borderline (BB) and borderline lepromatous (BL).

The collection of material for bacteriological examination and the determination of the bacterial index (BI) were performed by specialised professionals using the standard procedures recommended by the WHO (MS 2009). The slit skin smear test determined the BIs at diagnosis and at the end of treatment. Leprosy treatment followed WHO guidelines throughout the study.

The patients were classified as paucibacillary (PB), when the smear was negative, and as multibacillary (MB) when the smear was positive. The operational classification used in the routine treatment at the SAA is different from that used at other health care centres in Brazil, in which BIs are not considered and disease classification is based on the number of detected skin lesions.

For contact surveillance, the SAA maintains a cohort consisting of all the contacts of diagnosed patients, who are asked to attend the clinic for dermatological examination. Those with signs or symptoms of leprosy at this examination were evaluated via bacteriological and histopathological tests to confirm the diagnosis. The healthy contacts were instructed to return to the clinic if they later experienced any signs or symptoms of the disease.

The index leprosy patient was responsible for bringing his/her contacts to the clinic. If an MB patient was detected among the contacts after diagnosing a PB index case, the MB case was considered the index case. The contacts consisted of those who inhabited the same household as the patient or had close contact in other ways (such as neighbours and relatives) during the fiveyear period preceding diagnosis (MS 2010). Contacts were considered co-prevalent cases when leprosy was detected at initial examination. Incident cases did not have any signs or symptoms of the disease at the initial examination after an index case was diagnosed, but they were diagnosed during contact follow-up surveillance.

We selected 414 patients detected by contact surveillance at the SAA from 1987 through September 2010 among the cohort of contacts; 286 of the patients were co-prevalent cases and 128 were incident cases. Their respective index cases $(n=310)$, who were passively detected at the SAA during the same period, were selected for comparison.

Statistical analysis - The variables considered in this study were gender, age, clinical presentation, disability grade, $\mathrm{BI}$ at both diagnosis and the end of treatment and the presence of a reaction at diagnosis.

A conditional logistic regression model was used to estimate the odds ratios (ORs), taking into account the relationship between the contacts and their respective index cases (Hosmer \& Lemeshow 2000). Because of the close relationship between the contacts and their respective index cases, a similar approach was undertaken in a previous study conducted by our group (Sales et al. 2011), which examined the index-case factors associated with disease among contacts. The index cases were compared with both the co-prevalent and incident cases detected via contact surveillance. A significance level of $5 \%$ was established.

The variables that showed statistical significance in the bivariate analysis were considered for inclusion in the multivariate models. The final models consisted of the variables that remained significant in the multivariate models. Analyses were performed in Stata version 9.2 (StataCorp LP, College Station, Texas, USA).

Ethics - The Ethicals Committee of the Oswaldo Cruz Foundation approved the use of these data for research and publication in accordance with the Helsinki Declaration.

\section{RESULTS}

We analysed 286 co-prevalent and 128 incident cases detected via contact surveillance. These cases were related to 310 index cases of leprosy; 218 of the index cases (70.3\%) had one secondary case, $52(16.8 \%)$ had two secondary cases, $22(7.1 \%)$ had three secondary cases, 11 (3.5\%) had four secondary cases, four (1.3\%) had five secondary cases, one had six secondary cases and one had eight secondary cases.

All of the variables showed statistically significant differences $(p<0.001)$ in the bivariate analyses (Table I). While the majority of index cases were male (71.3\%), there were more females among the co-prevalent and incident cases detected by contact surveillance $(55.6 \%$ and $59.6 \%$, respectively). Among the index cases, $7.7 \%$ were under 15 years of age. Among the co-prevalent and incident cases, this proportion was much higher $(29.4 \%$ and $28.9 \%$, respectively). The predominant clinical presentations among the index cases were LL (46.5\%) and BL $(31 \%)$. In the cases detected by contact surveillance, the predominant clinical presentation was BT $(51.4 \%$ of the co-prevalent cases and $50 \%$ of the incident cases). The majority of index cases had MB leprosy (83.5\%), while most of the cases found through contact surveillance had the PB form ( $74.5 \%$ of the co-prevalent cases and $82.8 \%$ of the incident cases).

The percentages of index cases without disabilities at the beginning and end of treatment were $56.1 \%$ and $57 \%$, respectively. This percentage was much higher among the secondary cases $(82.7 \%$ and $86.4 \%$, respectively, of the co-prevalent cases and $87.4 \%$ and $86.5 \%$, respectively, of the incident cases). Reaction at diagnosis was present in $42.9 \%$ of the index cases, in $13.6 \%$ of the co-prevalent cases and in $7.8 \%$ of the incident cases.

The index cases had a higher initial bacterial index; namely, $34.7 \%$ of the index cases had a BI between 0-3 and $46.8 \%$ had a BI above 3 , while $73.7 \%$ of the co-prevalent and $83 \%$ of the incident cases had a BI of 0 .

In the simple logistic regression analyses, all of the ORs were statistically significant (Table II). The coprevalent contact cases were more likely to be female $(\mathrm{OR}=2.78)$ and 15 years of age or less $(\mathrm{OR}=5.44)$ and they were less likely to have the $\mathrm{MB}$ form $(\mathrm{OR}=0.01)$. In addition, compared with the index cases, participants were $72 \%$ less likely to have an initial disability of grade 
$1.81 \%$ less likely to have a final disability grade of 1 , $58 \%$ less likely to have an initial disability grade of 2 and $71 \%$ less likely to have a final disability grade of 2 .

The odds of reaction at diagnosis were $81 \%$ lower for co-prevalent cases detected by contact surveillance than for the passively detected cases. Compared with passively detected cases, co-prevalent cases also presented lower odds of having an initial and final BI of between 0 and 3 and they had even lower odds of having a BI above 3 .

Compared with the index cases, the incident cases were more likely to be female $(\mathrm{OR}=3.27)$ and 15 years of age or younger $(\mathrm{OR}=7.20)$, but they were less likely to have the MB form of the disease $(\mathrm{OR}=0.01)$.

Regarding disability at baseline, the incident cases detected by contact surveillance were $90 \%$ less likely to reach a disease grade of 1 and $70 \%$ less likely to reach grade 2 than the passively detected cases. Lower odds were also observed in the cases detected by contact surveillance with regard to reaching a final disability grade of $1(\mathrm{OR}=0.16)$ or $2(\mathrm{OR}=0.08)$. The odds of reaction at diagnosis were $90 \%$ lower for incident cases than for index cases. Incident cases also presented lower odds of

\section{TABLE I}

The clinical and epidemiological characteristics of the index cases, co-prevalent contacts and incident contacts, Souza Araújo Ambulatory patients, Rio de Janeiro, 1987-2010

\begin{tabular}{|c|c|c|c|}
\hline Variables $^{a}$ & $\begin{array}{l}\text { Index cases } \\
\mathrm{n}=310 \\
\mathrm{n}(\%)\end{array}$ & $\begin{array}{l}\text { Co-prevalent contacts } \\
\qquad \mathrm{n}=286 \\
\mathrm{n}(\%)\end{array}$ & $\begin{array}{c}\text { Incident contacts } \\
\mathrm{n}=128 \\
\mathrm{n}(\%)\end{array}$ \\
\hline \multicolumn{4}{|l|}{ Gender } \\
\hline Male & $221(71.3)$ & $127(44.4)$ & $52(40.6)$ \\
\hline Female & $89(28.7)$ & $159(55.6)$ & $76(59.4)$ \\
\hline \multicolumn{4}{|l|}{ Age (years) } \\
\hline$\leq 15$ & $24(7.7)$ & $84(29.4)$ & $37(28.9)$ \\
\hline$>15$ & $289(92.3)$ & $202(70.6)$ & $91(71.1)$ \\
\hline \multicolumn{4}{|l|}{ Clinical form } \\
\hline Tuberculoid leprosy & $0(0)$ & $4(1.4)$ & $6(4.7)$ \\
\hline Boderline tuberculoid & 37 (11.9) & $147(51.4)$ & $64(50)$ \\
\hline Borderline boderline & $19(6.1)$ & $19(6.6)$ & $10(7.8)$ \\
\hline Borderline lepromatous & $96(31)$ & $31(10.8)$ & $8(6.2)$ \\
\hline Lepromatous leprosy & $144(46.5)$ & $24(8.4)$ & $3(2.3)$ \\
\hline I & $7(2.3)$ & $43(15.1)$ & $27(21.1)$ \\
\hline NI & $2(0.6)$ & $17(5.9)$ & $8(6.2)$ \\
\hline NP & $5(1.6)$ & $1(0.3)$ & $2(1.5)$ \\
\hline \multicolumn{4}{|l|}{ Operational classification } \\
\hline Multibacillary & $259(83.5)$ & $73(25.5)$ & $22(17.2)$ \\
\hline Paucibacillary & $51(16.6)$ & $213(74.5)$ & $106(82.8)$ \\
\hline \multicolumn{4}{|l|}{ Initial disability grade } \\
\hline 0 & $170(56.1)$ & $229(82.7)$ & $104(87.4)$ \\
\hline 1 & $17(24.4)$ & $31(11.2)$ & $8(6.7)$ \\
\hline 2 & $59(19.5)$ & $17(6.1)$ & $7(5.9)$ \\
\hline \multicolumn{4}{|l|}{ Final disability grade } \\
\hline 0 & $150(57)$ & $178(86.4)$ & $83(86.5)$ \\
\hline 1 & $77(29.3)$ & $16(7.8)$ & $9(9.4)$ \\
\hline 2 & $36(13.7)$ & $12(5.8)$ & $4(7.1)$ \\
\hline \multicolumn{4}{|l|}{ Reaction at diagnosis } \\
\hline Yes & $133(42.9)$ & $39(13.6)$ & $10(7.8)$ \\
\hline No & $177(57.1)$ & $247(86.6)$ & $118(92.2)$ \\
\hline \multicolumn{4}{|l|}{ Initial bacterial index (BI) } \\
\hline 0 & $56(18.4)$ & $196(73.7)$ & $98(83)$ \\
\hline $0<\mathrm{BI} \leq 3$ & $106(34.7)$ & $42(15.8)$ & $12(10.2)$ \\
\hline$>3$ & $143(46.8)$ & $28(10.5)$ & $8(6.8)$ \\
\hline \multicolumn{4}{|l|}{ Final BI } \\
\hline 0 & $45(19.5)$ & $65(62.5)$ & $23(69.7)$ \\
\hline $0<\mathrm{BI} \leq 3$ & $134(58.3)$ & $29(27.9)$ & $7(21.2)$ \\
\hline$>3$ & $51(22.2)$ & $10(9.6)$ & $3(9.1)$ \\
\hline
\end{tabular}

$a$ : all the variables shown have statistically significant $(\mathrm{p}<0.001)$ differences when compared with the index cases. I: indeterminate leprosy; NI: nodular leprosy; NP: pure neural leprosy. 
having an initial or final BI of 0-3, while the odds were even lower of their BI being above 3. The clinical disease form, final BI and disability grade were not included in the final multivariate models due to the reduced number of observations in some of these categories and the lack of complete information for all of the cases. The operational classification was not included in the multivariate analysis because of co-linearity with the bacterial index. In the multivariate analysis of the incident cases, the BI remained statistically significant. For the co-prevalent cases among contacts, both age and BI were found to be statistically significant in the multivariate analysis (Table II).

\section{DISCUSSION}

For both the co-prevalent and incident cases diagnosed by contact surveillance, diagnosis occurred earlier in the progression of the disease and the disease had a less severe clinical presentation. These cases also exhibited lower levels of initial and final disability grades, a lower initial and final BI and a lower incidence of reac- tions. These differences were more significant between the prevalence and index cases than between the coprevalent and index cases.

For the most part, the sample group analysed in this study consists of individuals with difficult diagnoses, severe illnesses and other atypical features because the participants were from a national leprosy referral service. Because of this selection bias, this sample group does not constitute a representative sample of leprosy patients within the general population. However, the SAA is less prone to having operational problems, rigorously follows standard procedures and employs qualified professional staff. Another strength of this study is that these findings highlight the most recent trends in the leprosy epidemic and may be relevant in guiding subsequent interventions in the effort to control and prevent the spread of the disease.

Possibly due to inter-relationships among the variables, however, only the initial BI remained significant for the incident cases and only age and the initial BI remained

\section{TABLE II}

Odds ratios (ORs) (crude and adjusted) of the co-prevalent and incident contacts, Souza Araújo Ambulatory patients, Rio de Janeiro, 1987-2010

\begin{tabular}{|c|c|c|c|c|}
\hline Variables & $\begin{array}{c}\text { Crude OR } \\
\text { (CI 95\%) } \\
\text { co-prevalent contacts }\end{array}$ & $\begin{array}{c}\text { Adjusted OR } \\
\text { (CI 95\%) } \\
\text { co-prevalent contacts }\end{array}$ & $\begin{array}{c}\text { Crude OR } \\
\text { (CI 95\%) } \\
\text { incident contacts }\end{array}$ & $\begin{array}{c}\text { Adjusted OR } \\
\text { (CI 95\%) } \\
\text { incident contacts }\end{array}$ \\
\hline \multicolumn{5}{|l|}{ Gender } \\
\hline Male & 1 & 1 & 1 & 1 \\
\hline Female & $2.78(1.87-4.11)$ & $1.73(0.95-3.17)$ & $3.27(1.89-5.68)$ & $0.63(0.21-1.86)$ \\
\hline \multicolumn{5}{|l|}{ Age (years) } \\
\hline$\leq 15$ & $5.44(2.76-10.72)$ & $2.87(1.08-7.61)$ & $7.20(2.57-20.60)$ & $4.31(0.67-27.48)$ \\
\hline$>15$ & 1 & 1 & 1 & 1 \\
\hline \multicolumn{5}{|c|}{ Operational classification } \\
\hline Multibacillary & 1 & - & 1 & - \\
\hline Paubacillary & $0.01(0.01-0.08)$ & - & $0.01(0.01-0.08)$ & - \\
\hline \multicolumn{5}{|c|}{ Initial disability grade } \\
\hline 0 & 1 & 1 & 1 & 1 \\
\hline 1 & $0.28(0.16-0.49)$ & $0.83(0.39-1.79)$ & $0.10(0.03-0.29)$ & $0.79(0.17-3.68)$ \\
\hline 2 & $0.42(0.29-0.60)$ & $0.74(0.46-1.19)$ & $0.30(0.16-0.54)$ & $0.86(0.39-1.92)$ \\
\hline \multicolumn{5}{|c|}{ Final disability grade } \\
\hline 0 & 1 & - & 1 & - \\
\hline 1 & $0.19(0.09-0.39)$ & - & $0.16(0.06-0.43)$ & - \\
\hline 2 & $0.29(0.12-0.70)$ & - & $0.08(0.01-0.48)$ & - \\
\hline \multicolumn{5}{|c|}{ Reaction at diagnosis } \\
\hline Yes & $0.19(0.11-0.34)$ & $0.62(0.29-1.35)$ & $0.10(0.05-0.23)$ & $0.37(0.12-1.15)$ \\
\hline No & 1 & 1 & 1 & 1 \\
\hline \multicolumn{5}{|c|}{ Initial bacterial index (BI) } \\
\hline 0 & 1 & 1 & 1 & 1 \\
\hline $0<\mathrm{BI} \leq 3$ & $0.04(0.01-0.11)$ & $0.06(0.02-0.18)$ & $0.04(0.01-0.18)$ & $0.05(0.01-0.30)$ \\
\hline$>3$ & $0.02(0.01-0.06)$ & $0.04(0.01-0.12)$ & $0.01(0.01-0.07)$ & $0.02(0.01-0.14)$ \\
\hline \multicolumn{5}{|l|}{ Final BI } \\
\hline 0 & 1 & - & 1 & - \\
\hline $0<\mathrm{BI} \leq 3$ & $0.11(0.04-0.33)$ & - & $0.07(0.01-0.58)$ & - \\
\hline$>3$ & $0.12(0.03-0.53)$ & - & $0.06(0.01-1.25)$ & - \\
\hline
\end{tabular}

CI: confidence interval. 
significant among the co-prevalent cases. Again, taking into account the relationships among the clinical variables, the patients diagnosed through contact surveillance were diagnosed significantly earlier and had significantly lower bacterial loads compared with the index cases.

Recently published work by our group (Sales et al. 2011) using multilevel analyses measured the effect of index case characteristics on the risk of developing leprosy among contacts; this work showed that the bacillary load of the index case was the principle factor leading to disease among his/her contacts. The present study demonstrated that for the contacts and especially incident cases who became sick after the index case began treatment, there were reduced odds of disability and a severe clinical presentation, as well as increased odds of early diagnosis, in those who were detected through contact surveillance compared with passively detected cases. Reduced odds of final disability among the co-prevalent and incident cases detected among contacts were observed. However, importantly, this effect was not statistically significant in the final models. The disability grade has commonly been used as an indicator of diagnostic timeliness based on the assumption that the presentation of grades 1 and 2 constitutes evidence of late diagnosis.

Physical disability is considered the most severe complication of leprosy and the presence of reaction at diagnosis is a risk factor for developing disability (Saunderson 2000). In the present study, cases detected by contact surveillance presented lower odds of reaction at diagnosis.

Another study investigated the characteristics of the cases detected among the contacts between 1998-2002 and compared them with index cases. The results showed that the contact group had a higher proportion of female patients, children, skin lesions and nerve damage, but a greater proportion of negative smears, than the primary cases. In our results, however, there were no differences in the degrees of disability between the contact group and index cases (Ignotti et al. 2007).

The prevalence of the MB form of the disease may indicate delayed diagnosis. Our study showed higher odds of the paucibacillary form occurring among cases diagnosed by contact surveillance than among the passively detected index cases, thus confirming once again the importance of early diagnosis. Similarly, a study of 107 families conducted between 1998-2002 showed that the most frequent operational classification among contacts was $\mathrm{PB}$, but no age or gender differences between the co-prevalent and index cases were found (Duraes et al. 2010).

With respect to clinical form, the present study also found that the cases diagnosed by contact surveillance presented a less severe form of the disease. Likewise, Goulart et al. (2008) showed that in patients diagnosed between 2002-2007, the TT and BT clinical forms were predominant among contacts, but that LL was the most common among the index cases.

Different types of studies have confirmed contact surveillance as an important strategy in leprosy control. A microsimulation model developed to predict future leprosy trends showed that leprosy incidence would be substantially reduced by effective BCG vaccine coverage in concert with the combined strategies of contact tracing, early diagnosis and the treatment of infection and/or chemoprophylaxis in household contacts (Fischer et al. 2011).

On the other hand, Moet et al. (2004) noted that, despite epidemiological contact surveillance, transmission often occurs before index case detection. The results of the present study, however, reinforce the importance of contact surveillance in the prevention of leprosy.

A recent study demonstrated that, in endemic areas, neighbourhood contacts also appear to be important sources of transmission. Therefore, control measures in endemic areas should not be limited to households, but rather should include high-risk groups in the neighbouring areas of patient households as well (Feenstra et al. 2012).

Contact surveillance facilitates early diagnosis, which, in turn, helps control the spread of the disease, thus reducing the incidence of disability and diseaserelated stigma. Early detection immediately provides an opportunity for treatment, consequently leading to a reduction in contagiousness (Rodrigues \& Lockwood 2011). The introduction of multidrug therapy has led to a drastic decline in the prevalence of leprosy (Khubchandani 2011). Health education is also an important coadjuvant of prevention in contact surveillance strategies. Clearly, leprosy elimination activities, as detailed in the National Plan for Leprosy Elimination, should be expanded (MS 2006).

Leprosy in passively detected cases tends to be much more severe, thereby leading to the highest levels of disability and requiring larger investments in financial and human resources. Disabling injuries have a profound impact on the socio-economic sector of society and often lead to the exclusion of these patients from the labour force and even from social contact. A study delineating the impact of leprosy on quality of life found that leprosy causes high percentages of secondary injuries that compromise the capacity of the individual to engage in gainful employment and satisfactory life experiences and that perpetuate the stigma associated with the disease (Lustosa et al. 2011).

Government action for leprosy control should prioritise contact surveillance, as it has proven to be an indispensable tool in early leprosy detection and in preventing the more serious disease-related complications while also contributing to the control of disease spread.

In summary, the data in this study convincingly show that contact surveillance is a highly effective strategy in leprosy control efforts and that active surveillance is especially needed in highly endemic areas, such as Brazil.

\section{REFERENCES}

Düppre NC, Camacho LA, da Cunha SS, Struchiner CJ, Sales AM, Nery JA, Sarno EN 2008. Effectiveness of BCG vaccination among leprosy contacts: a cohort study. Trans $R$ Soc Trop Med Hyg 102: 631-638.

Duraes SMB, Guedes LS, Cunha MD, Magnanini MMF, Wand Del Rey de Oliveira ML 2010. Epidemiologic study of 107 cases of families with leprosy in Duque de Caxias, Rio de Janeiro, Brazil. An bras Dermatol 85: 339-345.

Feenstra SG, Nahar Q, Pahan D, Oskam L, Richardus JH 2012. Social contact patterns and leprosy disease: a case-control study in Bangladesh. Epidemiol Infect 14: 1-9. 
Fischer EA, de Vlas SJ, Habbema JD, Richardus JH 2011. The longterm effect of current and new interventions on the new case detection of leprosy: a modeling study. PLoS Negl Trop Dis 5: e1330.

Goulart IMB, Souza DOB, Marques CR, Pimenta VL, Gonçalves MA, Goulart LR 2008. Risk and protective factors for leprosy development determined by epidemiological surveillance of household contacts. Clin Vaccine Immunol 15: 101-105.

Hosmer DW, Lemeshow S 2000. Applied logistic regression, 2nd ed., John Wiley \& Sons, New York, 373 pp.

Ignotti E, Bayona M, Alvarez-Garriga C, Andrade VL, Valente JG 2007. Transmission of Hansen's disease and unscreened household contacts. Indian J Lepr 79: 11-25.

Khubchandani J 2011. State of the globe: many challenges of the multifaceted leprosy. J Glob Infect Dis 3: 315-316.

Lustosa AA, Nogueira LT, Pedrosa JI, Teles JB, Campelo V 2011.The impact of leprosy on health-related quality of life. Rev Soc Bras Med Trop 44: 621-626.

Mankar MJ, Joshi SM, Velankar DH, Mhatre RK, Nalgundwar AN 2011. A comparative study of the quality of life, knowledge, attitude and belief about leprosy disease among leprosy patients and community members in Shantivan leprosy rehabilitation centre, Nere, Maharashtra, India. J Glob Infect Dis 3: 378-382.

Moet FJ, Meima A, Oskan L, Richardus JH 2004. Risk factors for the development of clinical leprosy among contacts and their relevance for targeted interventions. Lepr Rev 75: 310-326.
MS - Ministério da Saúde 2002. Guia para o controle da hanseníase. [cited 2012 Nov 24]. Available from: bvsms.saude.gov.br/bvs/ publicacoes/guia_de_hanseniase.pdf.

MS - Ministério da Saúde 2006. Plano nacional de eliminação da hanseníase em nível municipal 2006-2010. [cited 2012 Jan 21]. Available from: portal.saude.gov.br/portal/arquivos/pdf/hanseniase_plano.pdf.

MS - Ministério da Saúde 2009. Guia de procedimentos técnicos para baciloscopia em hanseníase. [cited 2011 Apr 24]. Available from: portal.saude.gov.br/portal/arquivos/pdf/guia tecnico para baciloscopia_13_11_2009.pdf.

MS - Ministério da Saúde 2010. Portaria 3.125 de 7 de outubro de 2010. [cited 2011 Nov 24]. Available from: saude.es.gov.br/download/portaria_3125 hanseniase_2010.pdf.

Ridley DS, Jopling WH 1966. Classification of leprosy according to immunity. A five-group system. Int J Lepr 34: 255-273.

Rodrigues LC, Lockwood DN 2011. Leprosy now: epidemiology, progress, challenges and research gaps. Lancet Infect Dis 11: 464-470.

Sales AM, Ponce de Leon A, Düppre NC, Hacker MA, Nery JA, Sarno EN, Penna ML 2011. Leprosy among patient contacts: a multilevel study of risk factors. PLoS Negl Trop Dis 5: 1013.

Saunderson P 2000. The epidemiology of reactions and nerve damage. Lepr Rev 71 (Suppl.): S106-S110.

WHO - World Health Organization 2011. Leprosy update. Wkly Epidemiol Rec 36: 389-400. 